\title{
Screening of Dementia in Portuguese Primary Care: Methodology, Assessment Tools, and Main Results
}

\author{
Laetitia Teixeira ${ }^{1,2,3 *}$, Pedro Machado Dos Santos ${ }^{1,2}$, Sara Alves ${ }^{1}$, Maria João Azevedo ${ }^{1,2}$, \\ Mafalda Gomes Duarte ${ }^{1,2}$, António Leuschner ${ }^{1,4}$ and Constança Paúl ${ }^{1,2}$ \\ 1 UNIFAl, Instituto de Ciências Biomédicas Abel Salazar, Universidade do Porto, Porto, Portugal, ${ }^{2}$ CINTESIS, Instituto de \\ Ciências Biomédicas Abel Salazar, Universidade do Porto, Porto, Portugal, ${ }^{3}$ EPIUnit, Instituto de Saúde Pública, \\ Universidade do Porto, Porto, Portugal, ${ }^{4}$ Hospital Magalhães, Porto, Portugal
}

\section{OPEN ACCESS}

Edited by:

Matteo Cesari,

Centre Hospitalier Universitaire (CHU)

de Toulouse, France

Reviewed by:

Philipe De Souto Barreto,

Centre Hospitalier Universitaire (CHU)

de Toulouse, France

Mario Ulises Pérez-Zepeda, Instituto Nacional de Geriatría,

Mexico

Marco Inzitari,

Parc Sanitari Pere Virgili, Spain

*Correspondence:

Laetitia Teixeira

Icteixeira@icbas.up.pt

Specialty section:

This article was submitted to

Geriatric Medicine,

a section of the journal

Frontiers in Medicine

Received: 26 July 2017

Accepted: 27 October 2017

Published: 13 November 2017

Citation:

Teixeira L, Dos Santos PM, Alves S, Azevedo MJ, Duarte MG, Leuschner A and Paúl C (2017) Screening of Dementia in Portuguese

Primary Care: Methodology, Assessment Tools, and Main Results.

Front. Med. 4:197.

doi: 10.3389/fmed.2017.00197
The objectives of this article are as follows: (1) to describe the assessment protocol used to outline people with probable dementia in Primary Health Care; (2) to show the methodological design and procedure to obtain a representative sample of patients with probable dementia; and (3) to report the main characteristics of the sample collected in the context of the study "Characteristics and needs of people with probable dementia." The study protocol was based on the "Community Assessment of Risk and Treatment Strategies (CARTS) Program" and is composed by a set of instruments that allow the assessment of older adults with probable dementia in several areas (health, psychological, functionality, and other). Descriptive analysis was used to characterize the final sample $(n=436)$. The study protocol as well as the methodological procedure to obtain the referral of research participants and data collection on the condition of people with probable dementia in Primary Health Care proved to be a valuable tool to obtain a sample of patients distributed by the full range of probable dementia in a large geographical area. Results may allocate the design of care pathways for old people with cognitive disorders to prevent, delay impairment, and/or optimize quality of life of patients.

Keywords: caregivers, cognitive decline, dementia, old people, primary care

\section{INTRODUCTION}

The Portuguese Census 2011 (1) showed that in Portugal 19.1\% of the total population $(n=2,010,064)$ was aged 65 or plus. According some projections, this population will increase, with the group age $80+$ reaching the $15 \%$ in 2060 (2).

Presently little information is known in Portugal about the needs of the old people with probable dementia and their informal caregivers. Nunes et al. (3) estimated that the prevalence of cognitive impairment and dementia in Portuguese people living in the north of the country were 16.8 and $2.1 \%$ in rural areas, and 12 and $2.7 \%$ in urban areas, respectively; the majority of the reported cases were related with cerebrovascular diseases and vascular risk factors (48\%). A more recent study (4) revealed that the prevalence of dementia/Alzheimer's disease was 5.91\% in the Portuguese population with 60 or more years old. Considering the international context, the prevalence of dementia estimated by the World Health Organization (WHO) in South Europe increases with age, varying between $0.026 \%$ for individuals aged $65-69$ years and $0.324 \%$ for those aged $85+$ years (5). In addition, it will be expected an increase in the number of people with dementia, doubling by 2030 and tripling by 2050. These numbers have a high impact in the quality of life of the people and in the economy of the families and communities, representing one of the highest challenges/priorities of public health offices/professionals. 
Early detection of probable dementia is very important, and it appears to be under diagnosed by general practitioners (GPs). Nevertheless, GPs are well positioned to notice the possible cognitive decline of their patients and can be a major potential source for increasing the rate of case detection [e.g., Ref. $(6,7)]$. In a Finnish population based study, Lopponen et al. (8) found less than $50 \%$ of the patients with dementia with a diagnosis documented in primary care; the existence of diagnosis increased in more advanced stages of dementia.

High levels of poverty need to be considered in the topic of dementia (9-11) and must be addressed together with cultural aspects in the Portuguese context, namely, the low educational levels. This cross-sectional study has a main objective to draw a physical and mental health profile of the old people with dementia living in the north of Portugal and to understand their risk situation to further planning adequate responses and services for this specific population.

The main objectives of this article are as follows: (1) to describe the assessment protocol used to outline people with probable dementia in Primary Health Care; (2) to show the methodological design and procedure to obtain a representative sample of patients with probable dementia; and (3) to report the main characteristics of the sample collected in the context of the study "Characteristics and needs of people with probable dementia."

\section{MATERIALS AND METHODS}

\section{Participants}

The population of this study was defined as Portuguese people with 65 years and over, living in the community in the geographical area covered by the Portuguese North Regional Health Authority (ARS North) with mental health concerns. The geographical area is composed by 86 municipalities, which are organized in 24 Associations of Health Centres (ACES). The inclusion criteria were as follows: (a) outpatient of a health-care units integrated in an ACES covered by the ARS North and (b) age 65 or + years old. The exclusion criteria were as follows: (a) patient not using a primary health-care unit covered by the ARS North; (b) age less than 65 years old; (c) living in nursing home, hospital or psychiatric institution; and (d) absence of memory concerns [patients classified in stage 1 of the Global Deterioration Scale (GDS) $(12,13)]$.

\section{Sample}

Based on the distribution of Portuguese population with 65+ years old (1) and on the prevalence of dementia in the Western Europe predicted by the WHO (5), an estimate of Portuguese population with dementia by age groups is presented in Table $\mathbf{1}$.
The sample size, calculated for each age group, was considered as $1 \%$ of the estimated population with dementia. 572 participants with probable dementia compose the final sample. Table 1 the sample size calculation (total and by age group) according the prevalence of dementia.

\section{Measures}

The study protocol was based on the "Community Assessment of Risk and Treatment Strategies (CARTS) Program” developed in the University College Cork, Ireland (14). The study protocol includes instruments divided in three main parts: Part A: assessment of the patient with probable dementia; Part B: assessment of the patient with probable dementia by the health professional (GP or nurse); Part C: evaluation of the informal caregiver of the patient with probable dementia (if available). Table 2 resumes the domains evaluated and the instruments used in each part of the study protocol.

Mini-Mental State Examination $(15,16)$ is widely used for cognitive decline screening and is composed by 19 questions divided in 6 domains. The final score vary between 0 and 30 . GDS $(12,13)$ is used to classify individuals with cognitive decline according to a scale of seven points: 1. Without cognitive decline; 2 . Very mild cognitive decline; 3 . Mild cognitive decline; 4 . Moderate cognitive decline; 5. Moderately severe cognitive decline; 6. Severe cognitive decline; and 7. Very severe cognitive decline. $A B$ Clinician Depression Screen (17) is a brief version of the Geriatric Depression Scale and is composed by five dichotomist questions (yes/no). The final score vary between 0 and 5, and individuals with a score equal or higher to 3 present high probability of depression. Timed "Up and Go" (18) is a simple test used to assess a person's mobility, using the time that a person takes to rise from a chair, walk $3 \mathrm{~m}$, turn around, walk back to the chair, and sit down. Malnutrition Universal Screening Tool $(19,20)$ is a five-step screening tool to identify adults at risk of malnutrition or obese. The final score vary between 0 and 6 , considering three categories: 0 . Low risk; 1 . Moderate risk; and $\geq 2$. High risk. Short-Form Mini Nutritional Assessment $(21,22)$ is a valid nutrition screening and assessment tool that can identify patients who are malnourished or at risk of malnutrition and consist in six questions. The score vary between 0 and 14 and a score equal or higher to 11 is indicator of possible malnutrition. Bedside Swallow Assessment allows the evaluation of swallowing after sitting the people in a right posture and asking the person to drink $30 \mathrm{ml}$ of water. Three criteria were recorded and the final score of the test corresponds to the number of observed criteria: 1 . No criteria; 2 . Presence of 1 criterion; 3. Presence of 2 or more criteria. Handgrip strength is evaluated using a dynamometer considering four attempts, two in each hand. The final score correspond to the mean of the highest values. Exhaustion is evaluation considering a dichotomy

TABLE 1 | Sample size calculation (total and by age group) according the prevalence of dementia.

\begin{tabular}{|c|c|c|c|c|c|c|}
\hline & $65-69$ years & $70-74$ years & $75-79$ years & $80-84$ years & $85+$ years & Total \\
\hline Population & 180,352 & 150,687 & 136,275 & 97,113 & 72,399 & 636,826 \\
\hline Prevalence of dementia & 0.026 & 0.043 & 0.074 & 0.129 & 0.324 & - \\
\hline Estimated population with dementia & 4,689 & 6,480 & 10,084 & 12,528 & 23,457 & 57,238 \\
\hline Final sample (1\%) & 47 & 65 & 101 & 125 & 235 & 572 \\
\hline
\end{tabular}


TABLE 2 | Study protocol: domains and instruments used in each part.

\begin{tabular}{|c|c|c|}
\hline \multicolumn{3}{|l|}{ Part A } \\
\hline $\begin{array}{l}\text { A1. Sociodemographic } \\
\text { questionnaire }\end{array}$ & $\begin{array}{l}\text { Sex } \\
\text { Age } \\
\text { Education level } \\
\text { Profession } \\
\text { Marital status } \\
\text { Household } \\
\text { Residence context } \\
\text { Type of residence }\end{array}$ & $\begin{array}{l}\text { Infrastructures accessibilities } \\
\text { Formal care } \\
\text { Informal care } \\
\text { Use of health services } \\
\text { Medication } \\
\text { Health subsystem } \\
\text { Health expenditures }\end{array}$ \\
\hline A2. Cognition & \multicolumn{2}{|c|}{$\begin{array}{l}\text { Mini-Mental State Examination }(15,16) \\
\text { Global Deterioration Scale }(12,13)\end{array}$} \\
\hline A3. Depression & \multicolumn{2}{|c|}{ AB Clinician Depression Screen (ABCDS) (17) } \\
\hline A4. Biobehavioral aspects & \multicolumn{2}{|c|}{$\begin{array}{l}\text { Timed "Up and Go" (18) } \\
\text { Malnutrition Universal Screening Tool }(19,20) \\
\text { Short-Form Mini-Nutritional Assessment }(21,22) \\
\text { Bedside Swallow Assessment } \\
\text { Handgrip strength } \\
\text { Exhaustion } \\
\text { Physical activity } \\
\text { Tobacco and alcohol consumption } \\
\text { Whispered Voice Test (23) } \\
\text { Snellen Test (24) }\end{array}$} \\
\hline \multicolumn{3}{|l|}{ Part B } \\
\hline B1. Physical health & \multicolumn{2}{|c|}{ Older Americans Resources and Services $(25,26)$} \\
\hline B2. Adverse events & \multicolumn{2}{|c|}{ The Community Assessment of Risk Tool (14) } \\
\hline \multicolumn{3}{|l|}{ Part C } \\
\hline C1. Caregiver burden & \multicolumn{2}{|c|}{ Caregiver Burden Score (27) } \\
\hline C2. Depression & \multicolumn{2}{|l|}{$\operatorname{ABCDS}(17)$} \\
\hline C3. Mental health & \multicolumn{2}{|c|}{ Neuropsychiatric Inventory Questionnaire $(28,29)$} \\
\hline
\end{tabular}

question (yes/no) "In the last month, do you feel that you had very little energy to do the things you wanted to do?" Physical activity frequency evaluated using a four-point question: 1. >1/week; 2. 1/week; 3. 1-3/month; and 4. Almost never or never. Alcohol and tobacco consumption evaluated considering a set of questions about quantity, duration, and type. Whispered Voice Test (23) evaluate the audition and Snellen Test (24) the vision The physical health dimension of the Older Americans Resources and Services $(25,26)$ comprises a checklist of 16 diagnoses. The Community Assessment of Risk Tool-CART (14) evaluates the perceived risk of three adverse events: institutionalization, hospitalization and death. Caregiver Burden Score (27) assess the caregiver burden and score vary between 0 and 30. Score equal or higher to 15 is indicator of burden. Neuropsychiatric Inventory Questionnaire $(28,29)$ is a brief version of the Neuropsychiatric Inventory and allows the evaluation of psychopathology in dementia and its repercussion on the caregiver's overload. For each symptom, it evaluates the presence (yes/no), severity (1. Low; 2 . Moderate; and 3. Severe) and caregiver distress ( 0 . Not at all; 1 . Minimally; 2. Mildly; 3. Moderately; 4. Severely; and 5. Very severely or extremely).

\section{Ethical Procedure}

The study was submitted to the ethical committee of the ARS North-procedure number 6/2014 and approved at 7 January
2014. All the participants signed the informed consent form that was developed according the Declaration of Helsinki.

\section{Data Collection}

The data collection started in 2014 January and ended in 2016 April. Figure 1 shows the data collection's flowchart.

The first step consisted in the contact with the 24 ACES to obtain the authorization to do the study. All ACES had accepted to participate.

The second step consisted in contacting at least two healthcare units of each ACES with health professionals presenting interest in participating in the study. The health professional had as main responsibility filling in the screening instrument regarding the identification of people at risk of adverse health outcomes, namely, mental health concerns. The instrument used was the Risk Instrument for Screening in the Community (30), which is a new risk instrument for screening of old people. Based on the information about the patient, the health professional classified the patient in three different domains (mental health, ADLs, and physical/medical health) in a perceived risk scale (from 1 . Minimum risk to 5. Extreme risk) for the following three adverse events: hospitalization, institutionalization, and death. All health professional involved in this step received training to use this instrument by the investigators and the sessions took place in the health-care unit facilities. In this screnning, 55 health-care units were enrolled, with the participation of 285 health professionals who filled 7,298 valid screenings.

In the third step, and based on this screening and considering only patients with mental health concerns $(n=2,734)$, the sample was calculated using the stratified probability sampling method, considering sex, age groups, and ACES as strata. The technique used to extract patients for the sample was the lottery technique. Each patient with mental health concerns received a random number. The patients who received the higher numbers were invited to participate in the study, until all planned quotas were completed.

The health-care office contacted the selected patients, explaining the purpose of the study; if the patients agreed to participate they were further referred to the research team. In a second moment, the interviewers contacted the patients to schedule the interviews according to their availability. A limit of four contacts was fixed until a patient was withdrawn. In these situations, if available, another patient with similar conditions of the previous one was selected, according the sampling method described earlier.

The majority of the interviews were done in the health-care units (79.6\%), in an appropriate local where confidentiality was guaranteed. If it was impossible to do the interview in the health-care unit, the interviews were completed at patients' home $(19.9 \%)$. The main reason for the interviews to take place at home was the incapacity of the patient due to being bedridden or presenting low mobility. In the first moment of the interview, the patient was informed about the conditions of participation in the study, with the opportunity to clarify doubts. In order to formalize the interest of the patient in participating in the study, a personal Informative Consent was signed. If the patient did not have cognitive capacity, the signature of the consent was required to his/her legal representative. 


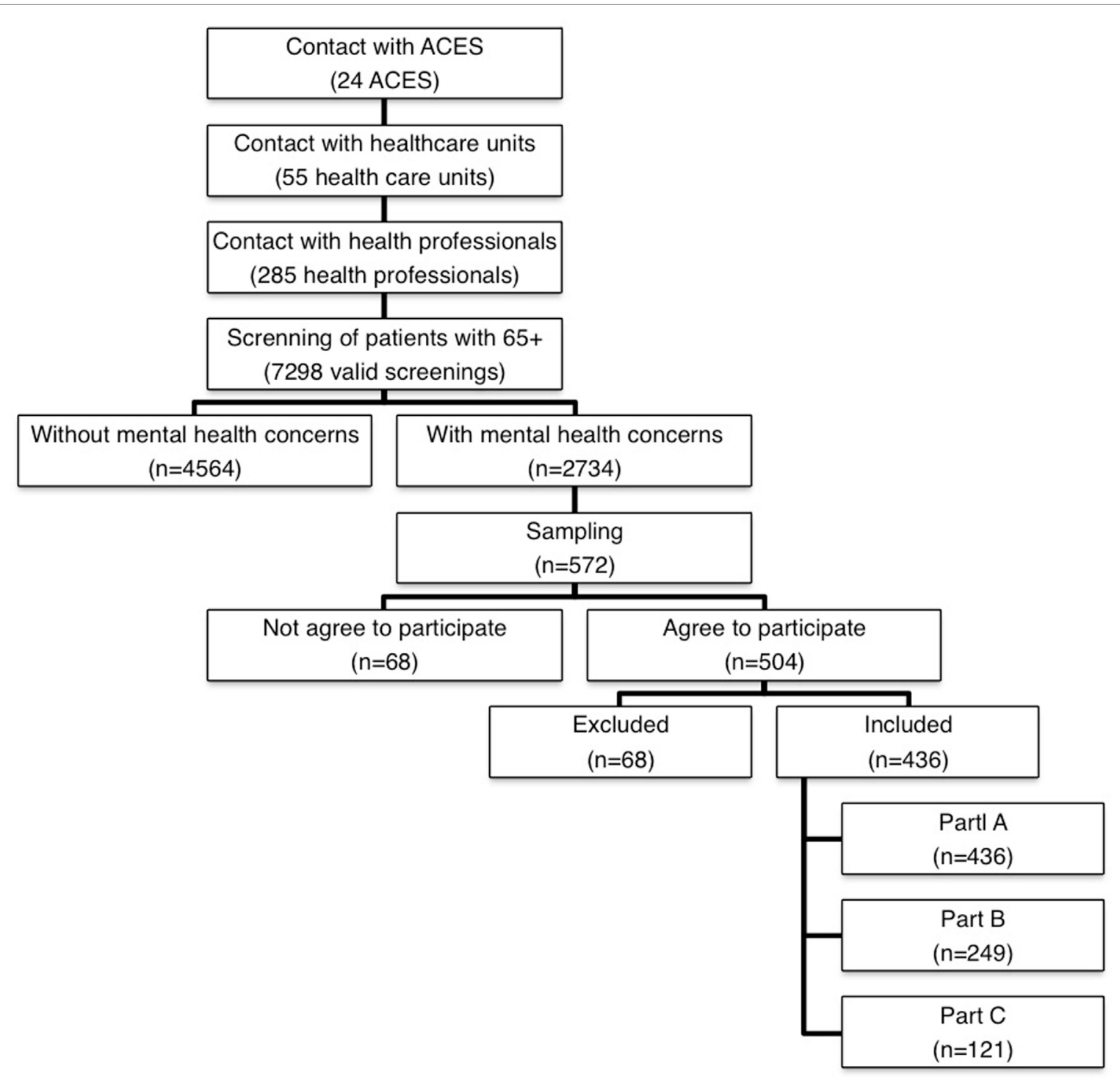

FIGURE 1 | Data collection's flowchart.

The study protocol took on average $45 \mathrm{~min}$ to complete. If the informal caregiver was present, the interviewer asked him/her to fill the Part C. After the interview, the health professional (GP or nurse) complete the Part B.

Regular meetings occur between the interviewers and the coordinator of the study with the purpose of supervising and monitoring of the data collection. The planning of data collection, the discussion of cases and the analysis of problems related with the scoring of the scales included in the study protocol were the main aspects discussed in the meetings.

The final sample comprised 436 patients with probable dementia. The ratio of execution was $76.2 \%$. The observed differences between the expected and collected samples are associated with some constrains related with the data collection, namely, difficulties/mistakes in the referral of cases and the high number of refuses to participate in the study (Figure 1).

\section{Statistical Analysis}

Given the presence of non-response related with the data collection, some groups were over- or underrepresented. A weighting adjustment procedure was implemented considering the projections of the population distributed by sex and age groups for 2012 (2). Descriptive analysis of the final weighted sample was performed to obtain a sociodemographic description of this population.

\section{RESULTS}

The sociodemographic characteristics of the sample $(N=436)$ are presented in Table 3. The sample included mostly women (58.7\%). The mean age was 75.2 years old ( $\mathrm{SD}=7.2$ years old). The education level with higher representation was primary level (1-4 years), and a relevant percentage of the sample was illiterate $(21.0 \%)$. Sixty-one percent were married/living with partner, $93.3 \%$ had children and 
TABLE 3 | Sociodemographic characteristics of the sample.

\begin{tabular}{|c|c|c|}
\hline Sociodemographic characteristics & $N$ & $\%$ \\
\hline Sex & 436 & \\
\hline Male & & 41.3 \\
\hline Female & & 58.7 \\
\hline Age & 436 & \\
\hline Years, mean (SD) & & $75.2(7.2)$ \\
\hline Education level & 434 & \\
\hline Illiterate & & 21.0 \\
\hline $1-4$ years & & 69.7 \\
\hline $5-6$ years & & 4.5 \\
\hline $7-9$ years & & 2.1 \\
\hline 10-12 years & & 1.9 \\
\hline$>12$ years & & 0.8 \\
\hline Marital status & 435 & \\
\hline Single & & 5.9 \\
\hline Married/lived with partner & & 60.9 \\
\hline Separated/divorced & & 4.5 \\
\hline Widowed & & 28.7 \\
\hline Children & 316 & \\
\hline No & & 6.7 \\
\hline Yes & & 93.3 \\
\hline$n$, mean (SD) & & $3.4(2.3)$ \\
\hline Grandchildren & 242 & \\
\hline No & & 16.2 \\
\hline Yes & & 83.8 \\
\hline$n$, mean (SD) & & $3.9(3.6)$ \\
\hline Living arrangement & 432 & \\
\hline Alone & & 15.7 \\
\hline Partner & & 62.9 \\
\hline Children & & 33.6 \\
\hline Other relative & & 21.2 \\
\hline Other & & 1.1 \\
\hline Context & 416 & \\
\hline Rural & & 47.9 \\
\hline Urban & & 52.1 \\
\hline
\end{tabular}

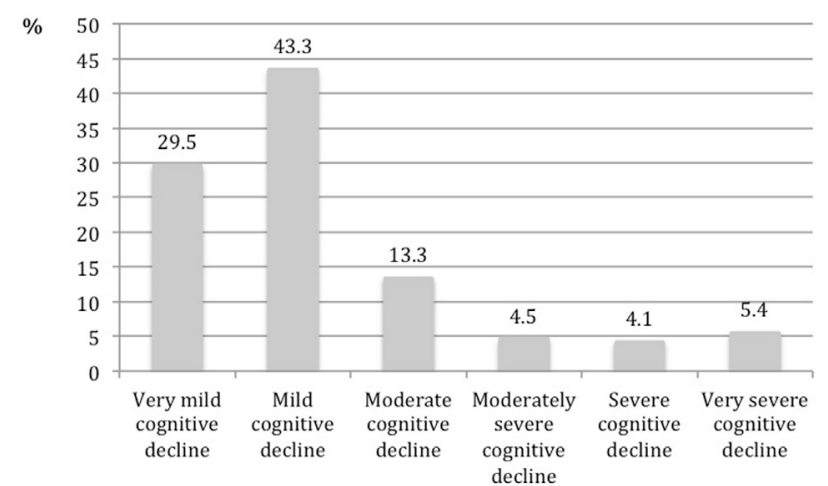

FIGURE 2 | Distribution of the sample according to the stage of the GDS scale.

$83.8 \%$ had grandchildren. The majority of the patients lived with a spouse or partner, with an expressive percentage living alone (15.7\%). The distribution of people by urban/rural contexts was balanced (52.1\% in urban areas and $47.9 \%$ in rural areas).

The distribution of the sample according to the stage of the GDS scale is presented in Figure 2. Forty-three percent of the sample was classified with mild cognitive decline, followed by $29.5 \%$ classified as very mild cognitive decline, and $13.3 \%$ as moderate cognitive decline. The most severe stages included $14 \%$ of the sample, with the stage "very severe cognitive decline" reaching $5.4 \%$. In the group of people evaluated by the GP $(N=249)$, $39 \%$ had a formal diagnosis of dementia.

\section{CONCLUSION}

The research design covering a large geographical area and the high participation of GPs in pre-screening patients from where the random sample was extracted are the main strengths of this study. The participation rate of GPs in the second phase of patients' assessment is the major limitation. The complex methodological process to obtain data on probable dementia patients in primary care, described earlier reflects the difficulty to tackle dementia in Primary Health-Care Services. Nevertheless, this procedure may configure a pathway of care that ultimately saves time and financial resources to GPs, preventing the comprehensive assessment of older patients that are not at risk of developing dementia.

The study protocol proved to be a valuable tool for a comprehensive assessment to identify patients and characterize their health needs and staging the cognitive decline. Based on GDS, the distribution of patients by different levels of probable dementia corroborate the findings of Lopponen et al. (8) and Prince et al. (31) for developed countries.

The enrollment of the primary health-care team and of the primary caregivers in the research facilitates the access to relevant data and mobilizes attention of professionals and family to an under diagnosis and under treated disease that leaves patients and carers helplessness.

It is barely feasible or adequate to assess every old adult for cognitive decline and we know that dementia seems to be reducing its prevalence at least in UK (32). Selecting people with mental health concern before sampling appears to be a good methodological approach to arrive to a clear distribution of patients across different stages of probable dementia. This will contribute to design effective pathways of care for people with cognitive decline. Mobilizing and training GPs and other primary care professionals will foster referral of patients to customized bundles of care, leading to a global and effective plan for dementia.

\section{ETHICS STATEMENT}

The study was submitted to the ethical committee of the ARS North-procedure number 6/2014 and approved at 7 January 2014. All the participants signed the informed consent form that was developed according the Declaration of Helsinki.

\section{AUTHOR CONTRIBUTIONS}

LT and CP conceived the research project design, wrote the manuscript, and conducted the data analysis. PS and AL contributed in the project conception and reviewed the manuscript. SA, MA, and $\mathrm{MD}$ were enrolled in the data collection and revised the manuscript.

\section{ACKNOWLEDGMENTS}

This work was supported by National Funds through the General Health Directorate of the Portuguese Ministry of Health. 


\section{REFERENCES}

1. INE. Censos 2011 Resultados Definitivos-Portugal. Lisboa, Portugal: Instituto Nacional de Estatística, IP (2012).

2. INE. Projecções de população residente em Portugal 2008-2060. Lisboa: Instituto Nacional de Estatística (2009).

3. Nunes T, Fragata I, Ribeiro F, Palma T, Maroco J, Cannas J, et al. The outcome of elderly patients with cognitive complaints but normal neuropsychological tests. J Alzheimer Dis (2010) 19:137-45. doi:10.3233/JAD-2010-1210

4. Santana I, Farinha F, Freitas S, Rodrigues V, Carvalho Á. The epidemiology of dementia and Alzheimer disease in Portugal: estimations of prevalence and treatment-costs. Acta Méd Port (2015) 28:182-8. doi:10.20344/amp.6025

5. WHO. Dementia. A Public Health Priority. United Kingdom: WHO (2012).

6. Ólafsdóttir M, Skoog I, Marcusson J. Detection of dementia in primary care: the Linköping study. Dement Geriatr Cogn Disord (2000) 11:223-9. doi:10.1159/000017241

7. Valcour VG, Masaki KH, Curb JD, Blanchette PL. The detection of dementia in the primary care setting. Arch Intern Med (2000) 160:2964-8. doi:10.1001/ archinte.160.19.2964

8. Lopponen M, Raiha I, Isoaho R, Vahlberg T, Kivela S-L. Diagnosing cognitive impairment and dementia in primary health care: a more active approach is needed. Age Ageing (2003) 32:606-12. doi:10.1093/ageing/afg097

9. Caamaño-Isorna F, Corral M, Montes-Martínez A, Takkouche B. Education and dementia: a meta-analytic study. Neuroepidemiology (2006) 26:226-32. doi:10.1159/000093378

10. Kalaria RN, Maestre GE, Arizaga R, Friedland RP, Galasko D, Hall K, et al. Alzheimer's disease and vascular dementia in developing countries: prevalence, management, and risk factors. Lancet Neurol (2008) 7:812-26. doi:10.1016/S1474-4422(08)70169-8

11. Sharp ES, Gatz M. The relationship between education and dementia an updated systematic review. Alzheimer Dis Assoc Disord (2011) 25:289. doi:10.1097/WAD.0b013e318211c83c

12. Grupo de Estudos de Envelhecimento Cerebral e Demência, GEECD. Escalas e testes na demência. 2nd ed. Lisboa: Novartis (2008).

13. Reisberg B, Ferris SH, de Leon MJ, Crook T. The global deterioration scale for assessment of primary degenerative dementia. Am J Psychiatry (1982) 139(9):1136-9.

14. O'Caoimh R, Healy E, Connell EO, Gao Y, Molloy DW. The community assessment of risk tool, (CART): investigation of inter-rater reliability for a new instrument measuring risk of adverse outcomes in community dwelling older adults. Ir J Med Sci (2012) 181(Suppl 7):227. doi:10.1007/s11845-012-0842-5

15. Folstein MF, Folstein SE, McHugh PR. "Mini-mental state": a practical method for grading the cognitive state of patients for the clinician. J Psychiatr Res (1975) 12:189-98. doi:10.1016/0022-3956(75)90026-6

16. Guerreiro M, Silva AP, Botelho MA, Leitão O, Castro-Caldas A, Garcia C. Adaptação à população portuguesa da tradução do Mini Mental State Examination (MMSE). Rev Port Neurol (1994) 1:9-10.

17. Molloy DW, Standish TI, Dubois S, Cunje A. A short screen for depression: the AB clinician depression screen (ABCDS). Int Psychogeriatr (2006) 18:481-92. doi:10.1017/S1041610205003078

18. Podsiadlo D, Richardson S. The timed "up \& go": a test of basic functional mobility for frail elderly persons. J Am Geriatr Soc (1991) 39:142-8. doi:10.1111/ j.1532-5415.1991.tb01616.x

19. Elia M. Screening for Malnutrition: A Multidisciplinary Responsibility. Development and Use of the Malnutrition Universal Screening Tool ('MUST') for Adults. Redditch: Worcs, BAPEN (2003).
20. Todorovic V, Russell C, Stratton R, Ward J, Elia M. The 'MUST' Explanatory Booklet: A Guide to the 'Malnutrition Universal Screening Tool' ('MUST') for Adults. Redditch: British Association for Parenteral and Enteral Nutrition (BAPEN) (2003).

21. Nestlé Nutrition Institute. Mini Nutritional Assessment MNA. (1994). Available from: http://www.mna-elderly.com/forms/MNA_portuguese.pdf

22. Rubenstein LZ, Harker JO, Salvà A, Guigoz Y, Vellas B. Screening for undernutrition in geriatric practice developing the short-form mini-nutritional assessment (MNA-SF). J Gerontol A Biol Sci Med Sci (2001) 56:M366-72. doi:10.1093/gerona/56.6.M366

23. MacPhee GJ, Crowther JA, McAlpine CH. A simple screening test for hearing impairment in elderly patients. Age Ageing (1988) 17:347-51. doi:10.1093/ ageing/17.5.347

24. Snellen H. Optotypi ad Visum Determinandum (Letterproeven tot Bepaling der Gezichtsscherpte; Probebuchstaben zur Bestimmung der Sehschaerfe). Utrecht, Netherlands: Weyers (1862).

25. Fillenbaum GG, Smyer MA. The development, validity, and reliability of the OARS multidimensional functional assessment questionnaire. JGerontol (1981) 36:428-34. doi:10.1093/geronj/36.4.428

26. Rodrigues RMC. Validação da versão em português europeu de questionário de avaliação funcional multidimensional de idosos. Rev Panam Salud Publica (2008) 23:109-15. doi:10.1590/S1020-49892008000200006

27. Zarit SH, Reever KE, Bach-Peterson J. Relatives of the impaired elderly: correlates of feelings of burden. Gerontologist (1980) 20:649-55. doi:10.1093/ geront/20.6.649

28. Ferreira A, Ribeiro O, Martins S, Fernandes L. Inventário Neuropsiquiátrico (NPI): Contributo para a Validação da Versão Portuguesa. Sinapse (2014) 14(2):152.

29. Kaufer DI, Cummings JL, Ketchel P, Smith V, MacMillan A, Shelley T, et al. Validation of the NPI-Q, a brief clinical form of the neuropsychiatric inventory. J Neuropsychiatry Clin Neurosci (2000) 12:233-9. doi:10.1176/ jnp.12.2.233

30. O’Caoimh R, Gao Y, Svendrovski A, Healy E, O'Connell E, O’Keeffe G, et al. Screening for markers of frailty and perceived risk of adverse outcomes using the risk instrument for screening in the community (RISC). BMC Geriatr (2014) 14:1. doi:10.1186/1471-2318-14-104

31. Prince M, Guerchet M, Prina M. The Global Impact of Dementia 2013-2050. London: Alzheimer's Disease International (2013).

32. Wu Y-T, Fratiglioni L, Matthews FE, Lobo A, Breteler MM, Skoog I, et al. Dementia in Western Europe: epidemiological evidence and implications for policy making. Lancet Neurol (2016) 15:116-24. doi:10.1016/S1474-4422 (15)00092-7

Conflict of Interest Statement: The authors declare that the research was conducted in the absence of any commercial or financial relationships that could be construed as a potential conflict of interest.

The reviewer PB and the handling editor declared their shared affiliation.

Copyright (C) 2017 Teixeira, Dos Santos, Alves, Azevedo, Duarte, Leuschner and Paúl. This is an open-access article distributed under the terms of the Creative Commons Attribution License (CC BY). The use, distribution or reproduction in other forums is permitted, provided the original author(s) or licensor are credited and that the original publication in this journal is cited, in accordance with accepted academic practice. No use, distribution or reproduction is permitted which does not comply with these terms. 